\title{
Hole and trion spin dynamics in quantum dots under excitation by a train of circularly polarized pulses
}

\author{
B. Eble, P. Desfonds, F. Fras, F. Bernardot, C. Testelin, and M. Chamarro \\ Institut des NanoSciences de Paris, UPMC Univ Paris 06, CNRS UMR 7588, 140 rue de Lourmel, 75015 Paris, France
}

A. Miard and A. Lemaître

Laboratoire de Photonique et Nanostructures, CNRS, Route de Nozay, F-91460 Marcoussis, France

(Received 6 October 2009; revised manuscript received 4 January 2010; published 29 January 2010)

\begin{abstract}
We have performed pump-probe experiments in $p$-doped InAs/GaAs quantum dots leading to the all-optical initialization and readout of hole spins. In order to describe these experiments, we have modelized the interconnected dynamics of the photoelectron spin and the resident hole spin, triggered through the optical excitation by a train of short pulses. A complete description of this spin dynamics is obtained by including the hyperfine coupling as the common decoherence mechanism for the electron and hole spins. Periodic excitation conditions for arbitrary values of the pump power and the external magnetic field are also included in the model. In particular, a good agreement concerning the temporal behavior of the photoinduced circular dichroism is obtained for zero or low magnetic fields. When the applied magnetic field screens the hole-hyperfine interaction, we show that the agreement between the experimental and calculated time-dependent curves requires an additional relaxation mechanism for holes with a characteristic time in the microsecond range.
\end{abstract}

DOI: 10.1103/PhysRevB.81.045322

PACS number(s): 72.25.Rb, 72.25.Fe, 78.67.Hc

\section{INTRODUCTION}

The spin of an electron confined in a quantum dot (QD) is a promising candidate for realizing a qubit in the solid state. Its optical initialization, readout, and manipulation have been the center of important investigations. ${ }^{1-8}$ At the same time, theoretical and experimental studies have shown that the hyperfine coupling with the lattice nuclei spins is the ultimate limit, at low temperature, to the electron-spin relaxation or decoherence in QDs, leading to typical ensemble dephasing times of the order of 1 ns in III-V QDs. ${ }^{9-12}$

Up to now, the studies have been centered on the spin of a conduction electron and only recently several experiments have evidenced the initialization and the readout of hole spins confined in QD nanostructures..$^{13-17}$ In particular, Gerardot et al. ${ }^{14}$ used a circularly polarized $\mathrm{cw}$ laser light to create a positively charged trion containing a polarized electron spin. This photocreated electron spin is coherently coupled via hyperfine interaction with the electron-spin projection which is not accessible by the optical excitation, so that the spontaneous emission of the trion states leads to the hole-spin polarization. The polarization of hole spins has also been observed under a periodic excitation of $p$-doped QDs by a train of circularly polarized short pulses. ${ }^{17}$ The same authors have then evidenced that the hyperfine interaction is, as for the electronic spin, at the origin of the holespin dephasing. The hyperfine interaction for hole spins is weaker than for electron spins due to the $p$-symmetry of the valence Bloch functions. This leads to a dipole-dipole character for the hole-nuclear-spin coupling instead of the Fermi contact character of the electron-nuclear-spin interaction. ${ }^{18,19}$

Here, we have performed and modelized pump-probe experiments leading to the initialization and readout of the hole spin in a $p$-doped QDs ensemble by optical excitation with a circularly polarized train of short pulses. In this situation, two kinds of spins have to be considered: the spin of the resident hole in the ground state and the spin of the photocreated three-particle complex, called trion, which is made of two holes and one electron. The spin of this positively charged trion is equal to the spin of its electron because its pair of holes is in the singlet state.

The interconnected dynamics of the resident hole and trion spins depend strongly on the excitation conditions (power of the excitation beam, pulsed or cw excitation, periodic or single-shot conditions), on the external conditions of evolution (free evolution or evolution in an external magnetic field), and on the efficiency and the nature of the relaxation mechanisms. The coupled dynamics of the resident electron and trion spins in pump-probe experimental configurations have been theoretically described for $n$-doped QDs in several previous works. In each of these studies, some simplifications have been performed and some aspects of the complete dynamics have been neglected. For example, a rather achieved description in presence and in absence of an external transverse magnetic field has been developed for a single-shot excitation. It takes into account "spontaneous" and "stimulated Raman coherence" whichever the excitation power, but it does not describe in detail the effect of the hyperfine relaxation mechanism on the electronic spins. ${ }^{20}$ The spontaneous decay of the trion state, by emission of a $\sigma+(\sigma-)$ polarized photon, leads in $n$-doped QDs to an electron spin polarized along the $+z(-z)$ direction, parallel to the growth axis, and thus generates quantum coherence between both Zeeman electron states in a magnetic field transverse to $z$. This effect has been called "spontaneously generated coherence." The conditions to insure a contribution to the electron coherence from the spontaneous emission of a trion are that the electronic Larmor frequency be comparable to, or smaller than, the trion decay rate and that the trion state be dipole coupled to both Zeeman electron states. A stimulated Raman coherence is also created by a $\sigma+(\sigma-)$ pump pulse in presence of a transverse magnetic field. The resonant 
$\sigma+(\sigma-)$ excitation of trion states in $n$-doped QDs leaves unperturbed the electron spins quantized in the $-z(+z)$ direction, which are superpositions of both spin states quantized in the magnetic field direction.

In a single-shot excitation description, Merkulov et al. ${ }^{10}$ took into account the nature of the electron-nuclear hyperfine interaction, which is modelized by the introduction of a frozen nuclear field whose the strength and direction vary from QD to QD. However, this theoretical work makes no description of the initialization step. The evolution of each individual electronic spin after its initialization is described as a precession around the total magnetic field, i.e., the nuclear field plus the external magnetic field. The decay of the average electron spin in the ensemble of QDs is a consequence of the random distribution of the local nuclear effective fields and it has a clear nonexponential behavior with a characteristic dephasing time $T_{\Delta}^{e}$. For standard QD sizes, $T_{\Delta}^{e} \approx 1 \mathrm{~ns}$.

Periodic excitation conditions were considered by Shabaev et $a .^{21}$ They considered a $\pi$-pulse excitation and the evolution of the electron spin in a transverse magnetic field, neglecting the spontaneous coherence. Later, a model of the periodic regime was developed by Greilich et al. ${ }^{22}$ for high transverse magnetic fields, neglecting the spontaneous coherence while studying the effect of the pump area. More recently, the same group has completed the description by adding spontaneous coherence. ${ }^{23}$ None of the cited works, which have considered a periodic excitation, took into account the singular time evolution of the electron spin due to its interaction with the surrounding nuclei.

In this work, in order to describe recent time-resolved experiments in $p$-doped QDs leading to the all-optical initialization and readout of hole spins, we developed a description of the interconnected dynamics of the photocreated electron spins and the resident hole spins taking into account the hyperfine coupling as the main and common mechanism responsible for the spin decoherence. Moreover, we considered the periodicity of the optical excitation for any value of the pump power, at zero or nonzero value of an applied magnetic field in the Faraday geometry. As other authors, we have used the optical selection rules of a pure heavy hole (hh) in spite of the fact that the hole mixing has made possible the first experimental evidence of the hole-hyperfine interaction in QDs. ${ }^{17}$ Indeed, hole mixing is at the origin of hole-spin dephasing via the hole hyperfine interaction, but remains a second-order perturbation to the optical selection rules even in a Rabi excitation regime near $\pi$-pulse. Finally, our model includes a contribution to the hole-spin populations induced by the trion emission.

\section{SAMPLE AND PUMP-PROBE EXPERIMENTS}

We have studied a structure grown by molecular-beam epitaxy on a (001) GaAs substrate. It consists of 30 planes of InAs self-assembled QDs, separated by 38-nm-thick GaAs spacer layers. The area QD density is about $10^{10} \mathrm{~cm}^{-2}$. The structure was $p$-modulation doped with a Carbon deltadoping layer (nominal density $\sim 2.10^{11} \mathrm{~cm}^{-2}$ ) located below each QD layer. Figure 1(a) shows the photoluminescence (PL) spectrum obtained at low temperature $(2 \mathrm{~K})$ with an excitation of the sample at $1.43 \mathrm{eV}$.
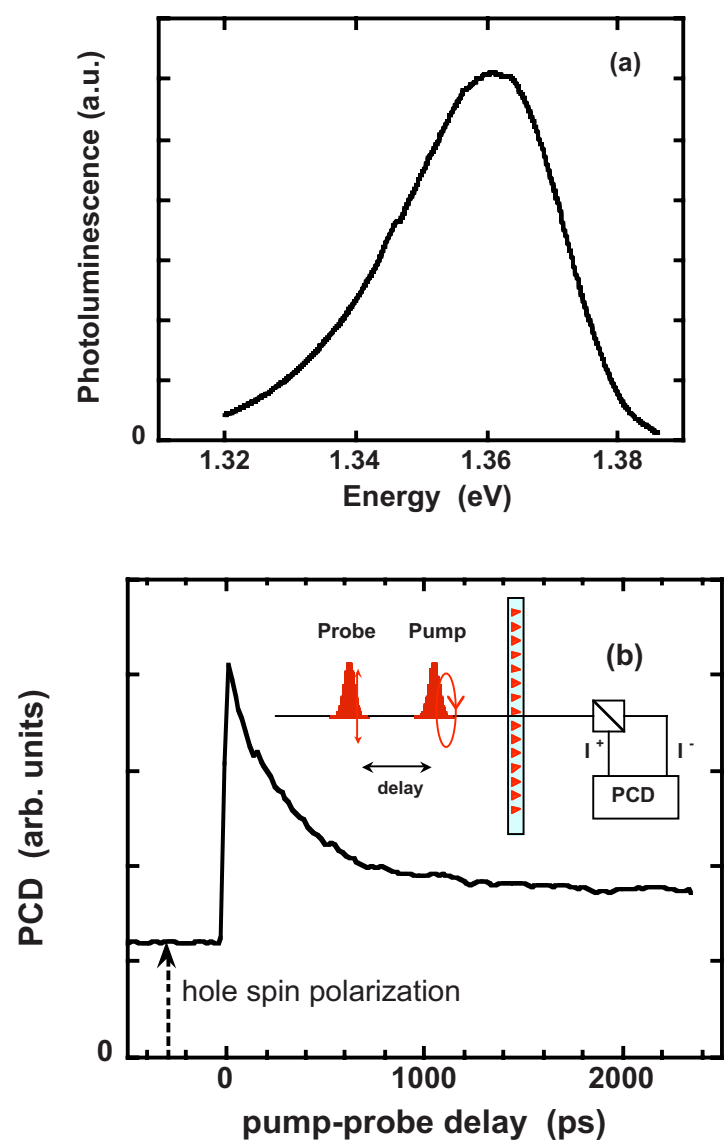

FIG. 1. (Color online) (a) Low-temperature (2 K) PL spectrum of the $p$-doped InAs/GaAs QDs. The sample is made of 30 planes of QDs with surface density $10^{10} \mathrm{~cm}^{-2}$. The excitation energy is $1.43 \mathrm{eV}$. (b) Low-temperature (2 K) PCD signal vs pump-probe delay at zero magnetic field. The pump and probe energies are tuned at the lowest excited state of the $p$-doped InAs/GaAs QDs $(E$ $=1.35 \mathrm{eV})$. The polarization of the pump beam is $\sigma^{+} / \sigma^{-}$modulated at $42 \mathrm{kHz}$. The pump power density is $\approx 60 \mathrm{~W} \mathrm{~cm}^{-2}$. (Inset) Schematic representation of the PCD measurement.

We have performed pump-probe experiments on this sample, the optical beams propagating along the growth axis. The photoinduced circular dichroism (PCD) has been measured by using a picosecond Ti:sapphire laser tuned at 1.35 $\mathrm{eV}$, which is split into the pump and probe beams (the repetition frequency is $76 \mathrm{MHz}$ ). The pump beam polarization is $\sigma+/ \sigma-$ modulated at $42 \mathrm{kHz}$ with a photoelastic modulator and the probe beam is linearly polarized. After transmission through the sample, the probe beam is decomposed into its two circular components and the difference of their intensities is measured with a balanced optical bridge [see inset of Fig. 1(b)]. To improve the signal-to-noise ratio, a double lock-in amplifier analysis of the signal is performed, the pump and probe beams being modulated with a mechanical chopper at two different frequencies.

Figure 1(b) shows the temporal behavior of the lowtemperature PCD signal obtained when the pump and probe beams are tuned at the trion transition of the $p$-doped QDs. Considering pure hh states, the PCD signal has two contributions: (i) the population difference $\rho_{+3 / 2}-\rho_{-3 / 2}$ of the spinpolarized hh ground states $J_{z}^{h}= \pm 3 / 2\left(\rho_{ \pm 3 / 2}\right.$ being the popu- 
lation of hole state $\pm 3 / 2$ ) and (ii) the population difference $\rho_{+1 / 2}-\rho_{-1 / 2}$ of the spin-polarized trion states $S_{z}= \pm 1 / 2$ $\left(\rho_{ \pm 1 / 2}\right.$ being the population of electron state $\left.\pm 1 / 2\right)$. Thus the PCD signal writes $\operatorname{PCD} \propto\left(\rho_{+3 / 2}-\rho_{-3 / 2}\right)-\left(\rho_{+1 / 2}-\rho_{-1 / 2}\right)$. In Fig. 1(b), we observe a nonzero PCD signal at negative pump-probe delays, indicating that the spin polarization is not fully relaxed within the $T_{L}=13$ ns repetition period of the laser pulses. This long-living component of the PCD signal is unambiguously associated to the net spin polarization of the resident holes, the only species present in the sample after the radiative recombination of the trions $\left[T_{R} \approx 800 \mathrm{ps}\right.$ (Ref. 17)]. Similar results were obtained in a set of samples with different $p$-doping contents (from 0.8 to $2 \times 10^{11} \mathrm{~cm}^{-2}$ ). The long-living PCD signal increases as $p$-doping increases. We have centered here our study on the sample containing the higher doping level, for which we have determined experimentally that most of QDs contain a single resident hole. $^{24}$

In first approximation, when pure hh states are considered, the spin polarization of the resident holes in the QDs, built by a pulsed excitation resonant with the trion optical transition, is explained as follows: a circularly polarized pump beam, propagating along $z$ and tuned at the energy of the lowest-allowed optical transition of InAs QDs containing a single resident $\mathrm{hh}$, creates a complex of three particles, called the positively charged trion $\left(X^{+}\right)$, in its ground state. The spin of the photogenerated electron points down or up depending on the $\sigma^{+}$or $\sigma^{-}$circularly polarized excitation, respectively. Thus, the pump beam helicity selectively generates spin-polarized electrons. During the lifetime of these photogenerated electrons, the efficient electron-nuclear hyperfine interaction leads to a coherent coupling of their spin $z$ components in each QD. The spontaneous decay of the trion states by emission of polarized photons then leads to an efficient hole-spin cooling. ${ }^{14,17}$

\section{MODEL OF HOLE AND TRION SPINS DYNAMICS}

We have considered the dynamics, in $p$-doped QDs, of the resident hole spin and the photocreated electron spin under the periodic excitation by an infinite train of $\sigma^{+}$circularly polarized pump pulses, propagating along the $z$ direction, with period $T_{L}$. We are interested in modelizing the temporal behavior of the PCD signal, equal to the difference of the photoinduced absorption for the $\sigma^{+}$and $\sigma^{-}$components of a probe beam:

$$
\begin{aligned}
\operatorname{PCD}(t)= & \alpha_{\sigma-}^{P}(t)-\alpha_{\sigma+}^{P}(t) \propto\left[\rho_{+3 / 2}(t)-\rho_{-3 / 2}(t)\right] \\
& -\left[\rho_{\uparrow}^{e}(t)-\rho_{\downarrow}^{e}(t)\right],
\end{aligned}
$$

where $\alpha_{\sigma+}^{P}(t)\left[\alpha_{\sigma-}^{P}(t)\right]$ is the absorption of a $\sigma^{+}\left(\sigma^{-}\right)$probe photoinduced by the $\sigma^{+}$pump pulse, $\rho_{+3 / 2}(t)\left[\rho_{-3 / 2}(t)\right]$ denotes the population of the heavy hole $+3 / 2 \Uparrow(-3 / 2 \downarrow)$, and $\rho_{\uparrow}^{e}(t)\left[\rho_{l}^{e}(t)\right]$ represents the population of the trion state containing a spin-up electron $\uparrow \Downarrow \Uparrow$ (a spin-down electron $\downarrow \Downarrow \Uparrow$ ). Then the PCD signal (1) can also be written as

$$
\operatorname{PCD}(t) \propto \frac{2 J_{z}(t)}{3}-2 S_{z}(t) .
$$

While the pump pulse, with its 2-ps duration, interacts with the QDs, we neglect any other process. For pure hh states, a $\sigma^{+}$pump operates a Rabi oscillation with a transfer factor $\sin ^{2}(\Theta / 2)$ between the hole state $\Downarrow$ and the trion state $\downarrow \Downarrow \Uparrow$ (see Fig. 2). The pump power appears through the pulse area $\Theta=\int d E(t) d t / \hbar$, where $d$ is the dipolar matrix element of the transition and $E(t)$ is the envelope of the pump optical field.

The $z$ component of the photocreated electron spin $\vec{S}\left(0^{+}\right)$, after a $\sigma^{+}$pump excitation with a pulse area $\Theta$, has the form

$$
S_{z}\left(0^{+}\right)=\frac{A}{2}=\frac{1}{4} \sin ^{2}(\Theta / 2)\left(\frac{2}{3} J_{z}\left(0^{-}\right)-1\right) .
$$

$t=0^{+}\left(0^{-}\right)$corresponds to the QD state just after (before) the pump pulse. Once the electron and hole spins have been initialized by a short pump pulse, we assume that the main decoherence mechanism for the spins of electrons and holes is the hyperfine interaction, which has a different nature for electrons ${ }^{9,10}$ and holes. ${ }^{17-20}$ For electrons, the Fermi contact term leads to an isotropic interaction, meanwhile for holes, the dominant term is the dipole-dipole term that leads to an anisotropic interaction. In both cases, the hyperfine interaction can be modelized by a frozen effective nuclear field whose the strength and direction vary from QD to QD. This nuclear field is denoted $\vec{B}_{N}^{e}$ for electrons and $\vec{B}_{N}^{h}$ for holes. In the absence of an external magnetic field, each spin in each QD precesses coherently around the nuclear field and the average hole or electron spin polarization in the QD ensemble decays partially with time as a consequence of the random distribution of the local nuclear effective fields from QD to QD. Figure 2 represents a scheme of the photoelectron and hole-spins evolution in the absence of an external magnetic field. The corresponding ensemble dephasing times are denoted in the following by $T_{\Delta}^{e}$ and $T_{\Delta}^{h}$, respectively, for the electron and hole spins.

The period, $T_{L}=13 \mathrm{~ns}$, of the pump pulses being short compared to the typical fluctuation time of the nuclear spins, we can consider that a periodic time dependence is established for the trion and hole-spin polarizations, in presence of a frozen nuclear field. In a single QD, the resident hole spin $\vec{J}(t)$ [respectively, the photocreated electron spin $\vec{S}(t)]$ is submitted to a nuclear field $\vec{B}_{N}^{h}$ (respectively, $\vec{B}_{N}^{e}$ ) and to a magnetic field $\vec{B}$ applied along the $z$ axis (Faraday configuration). For simplicity, we have considered a constant envelope wave function for the hole and the electron inside a QD. The correlation between $\vec{B}_{N}^{h}$ and $\vec{B}_{N}^{e}$ has been included by fixing the following relations between the in-plane (II subscribe) and out-of-plane components ( $z$ subscribe):

$$
\frac{B_{N_{\|}}^{h}}{B_{N_{\|}}^{e}}=\frac{2|\beta| B_{N z}^{h}}{\sqrt{3} B_{N z}^{e}}=\frac{2|\beta|}{\sqrt{3}\left(1+|\beta|^{2}\right)} \frac{g_{e}}{g_{h}} \frac{T_{\Delta}^{e}}{T_{\Delta_{0}}^{h}}=\frac{g_{e}}{g_{h}} \frac{T_{\Delta}^{e}}{T_{\Delta}^{h}},
$$

where $|\beta|$ fixes the mixed character of the hh state and is defined in Ref. 19; $T_{\Delta_{0}}^{h}$ is the ensemble decoherence time for pure hh states. ${ }^{19} g_{h}$ and $g_{e}$ are the Landé factor along $z$ for hole and electron, respectively. The above relations are exact in presence of a single nuclear species. In the presence of 


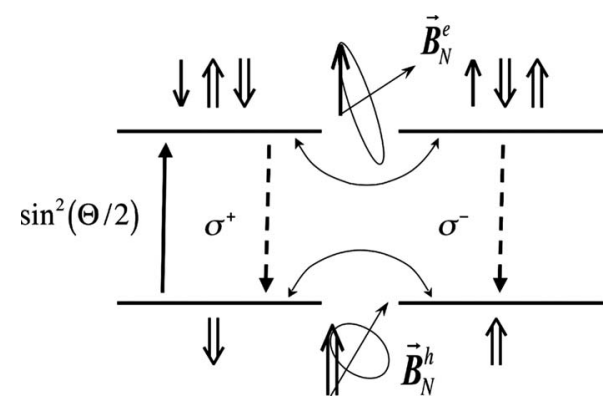

FIG. 2. A $\sigma^{+}$pump pulse drives Rabi oscillations between the $\Downarrow$ hole state and the $\downarrow \Uparrow \Downarrow$ trion state, with a population transfer factor $\sin ^{2}(\Theta / 2)$, where $\Theta$ is the pump pulse area (solid arrow). The hyperfine interaction of an electron (a hole) with the nuclear spins coherently couples both z-projection of spin states of the trion (hole) within a QD. In the mean-field approximation, the effect of the nuclei on the trion (hole) spin is described by the electron (hole) spin precession in an effective magnetic field $\vec{B}_{N}^{e}\left(\vec{B}_{N}^{h}\right)$ which has random direction and modulus from $\mathrm{QD}$ to $\mathrm{QD}$. As a consequence of the randomness of these Overhauser fields, the average trion (hole) spin polarization in the ensemble of QDs decreases partially as function of time, making possible both $\sigma^{+} / \sigma^{-}$emissions (dashed arrows).

two different species, a more careful study of the correlation between both nuclear fields should be needed. However, both fields being mainly due to the In nuclei (because of the large value of $I_{\text {In }}=9 / 2$ ), we kept the relations (4) between the nuclear fields felt by a hole and by a trion.

The $x^{\prime}$ axis is chosen such that the nuclear fields are within the $z x^{\prime}$ plane and are defined by the components $\left(B_{N}^{e}\right.$, $\left.\xi_{e}, \varphi_{e}=\varphi_{h}\right)$ and $\left(B_{N}^{h}, \xi_{h}, \varphi_{h}\right)$ in spherical coordinates. When an external magnetic field is applied in Faraday configuration, the electron spin $\vec{S}(t)$ precesses around the total magnetic field $\vec{B}_{N}^{e}+\vec{B}$, with pulsation $\Omega_{e}$, and the hole spin $\vec{J}(t)$ around the total magnetic field $\vec{B}_{N}^{h}+\vec{B}$, with pulsation $\Omega_{h}$. Because of the periodicity of the pump pulses, the hole and electron spins satisfy $\vec{J}\left(t+T_{L}\right)=\vec{J}(t)$ and $\vec{S}\left(t+T_{L}\right)=\vec{S}(t)$ in the stationary regime.

In a magnetic field $\vec{B}$ applied along $z$, the electron spin then precesses around $\vec{B}_{N}^{e}+\vec{B}$ of components $\left(\left|\vec{B}_{N}^{e}+\vec{B}\right|, \widetilde{\xi}_{e}, \varphi_{h}\right)$ and recombines with the trion lifetime $T_{R}$. We assume that the dynamics of the electron spin writes

$$
\frac{d \vec{S}(t)}{d t}=\frac{g_{e} \mu_{B}}{\hbar}\left[\left(\vec{B}+\vec{B}_{N}^{e}\right) \times \vec{S}(t)\right]-\frac{\vec{S}(t)}{T_{R}} .
$$

Solving Eq. (5), we obtain the time dependence

$$
\left\{\begin{array}{l}
S_{x^{\prime}}(t)=A \frac{1}{2} \sin \widetilde{\xi}_{e} \cos \widetilde{\xi}_{e}\left[1-\cos \Omega_{e} t\right] \exp \left(-t / T_{R}\right) \\
S_{y^{\prime}}(t)=-A \frac{1}{2} \sin \widetilde{\xi}_{e} \sin \Omega_{e} t \exp \left(-t / T_{R}\right) \\
S_{z}(t)=A \frac{1}{2}\left[\cos ^{2} \widetilde{\xi}_{e}+\sin ^{2} \widetilde{\xi}_{e} \cos \Omega_{e} t\right] \exp \left(-t / T_{R}\right),
\end{array}\right.
$$
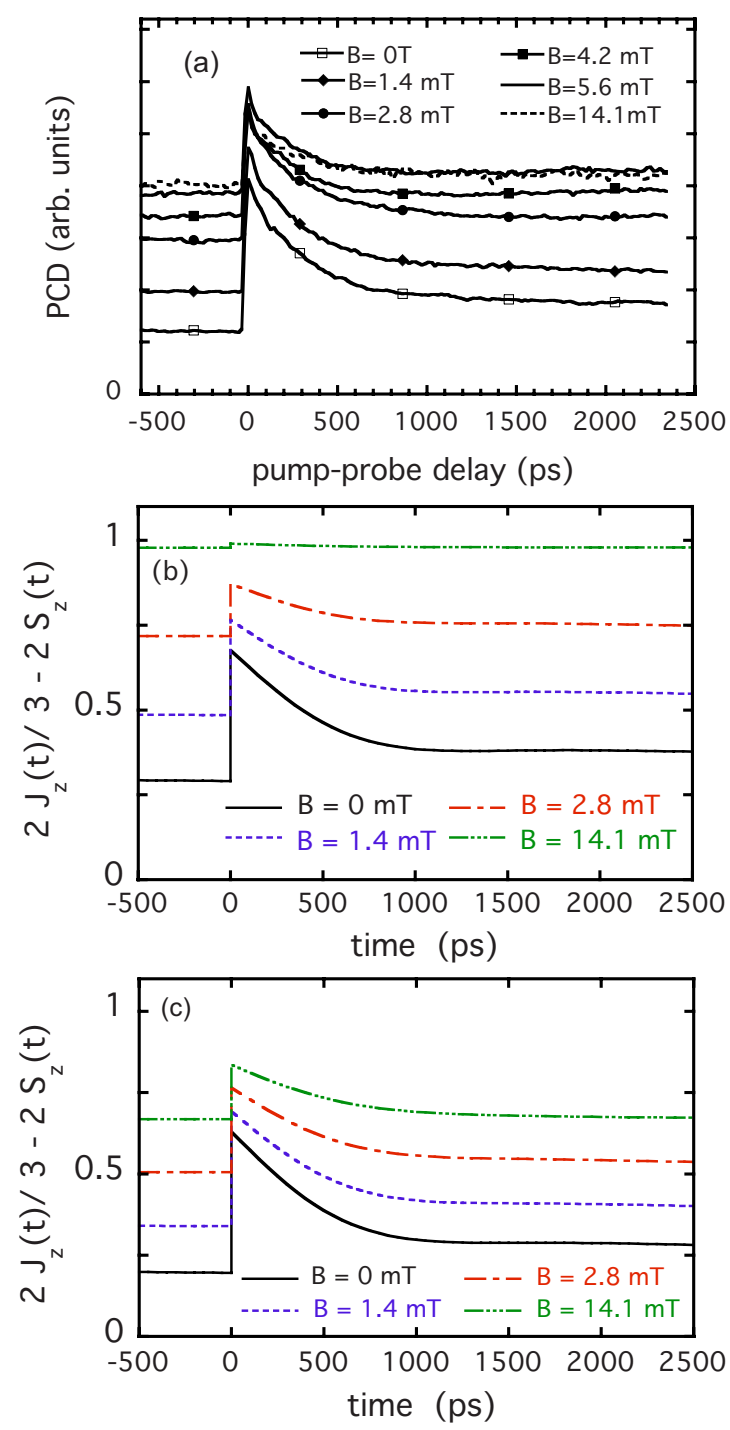

FIG. 3. (Color online) (a) Experimental PCD signal vs pumpprobe delay for different values of the external magnetic field $\vec{B}$ applied in a Faraday configuration (i.e., along the sample growth axis $z$ ). The pump and probe beams are tuned at $1.35 \mathrm{eV}$. The zero-signal level is the same for all displayed curves. $T=2 \mathrm{~K}$. (b) Calculated PCD signal vs pump-probe delay for different values of the external Faraday magnetic field $\vec{B}$. The only source of spin relaxation is supposed to be the hyperfine interaction. (c) Calculated PCD signal with the additional relaxation time $T_{1}=0.3 \mu$ s for hole spins. In (b) and (c), we have used the following values: $T_{R}$ $=780 \mathrm{ps}, T_{\Delta}^{e}=550 \mathrm{ps}, g_{e}=-0.4, g_{h}=1.35, \Theta=1.7,|\beta|=0.42$, and $T_{\Delta_{0}}^{h}=4$ ns.

with $\hbar \Omega_{e}=g_{e} \mu_{B}\left|\vec{B}_{N}^{e}+\vec{B}\right|$.

We assume that the dynamics of the hole spin is connected with the trion spin dynamics by the equation

$$
\frac{d \vec{J}}{d t}=\frac{g_{h} \mu_{B}}{\hbar}\left[\left(\vec{B}+\vec{B}_{N}^{h}\right) \times \vec{J}\right]+\frac{3(\vec{S} \cdot \vec{z}) \vec{z}}{T_{R}},
$$

where $\vec{z}$ is the unit vector along $z$ axis. When a trion relaxes 
by emitting a photon, it induces a spontaneous hole spin $\vec{J}_{1}(t)$. During the time interval $\left[t^{\prime}, t^{\prime}+d t^{\prime}\right]$, this spontaneous emission generates a change of hole spin

$$
d\left[\frac{2 J_{1 z}\left(t^{\prime}\right)}{3}\right]=\left[2 S_{z}\left(t^{\prime}\right)\right] \frac{d t^{\prime}}{T_{R}},
$$

where $2 S_{z}=\rho_{+1 / 2}-\rho_{-1 / 2}$ is the difference between the populations of electron states $\pm 1 / 2$. Afterwards, $d \vec{J}_{1}$ then precesses around $\vec{B}_{N}^{h}+\vec{B}$ of components $\left(\left|\vec{B}_{N}^{h}+\vec{B}\right|, \widetilde{\xi}_{h}, \varphi_{h}\right)$. The summation of all the contributions during the interval $[0, t]$ then gives

$$
\left\{\begin{array}{l}
J_{1 x^{\prime}}(t)=\frac{3}{2} \int_{0}^{t} 2 S_{z}\left(t^{\prime}\right) \sin \widetilde{\xi}_{h} \cos \widetilde{\xi}_{h}\left[1-\cos \Omega_{h}\left(t-t^{\prime}\right)\right] \exp \left[-\left(t-t^{\prime}\right) / T_{R}\right] \frac{d t^{\prime}}{T_{R}} \\
J_{1 y^{\prime}}(t)=-\frac{3}{2} \int_{0}^{t} 2 S_{z}\left(t^{\prime}\right) \sin \widetilde{\xi}_{h} \sin \Omega_{h}\left(t-t^{\prime}\right) \exp \left[-\left(t-t^{\prime}\right) / T_{R}\right] \frac{d t^{\prime}}{T_{R}} \\
J_{1 z}(t)=\frac{3}{2} \int_{0}^{t} 2 S_{z}\left(t^{\prime}\right)\left[\cos ^{2} \widetilde{\xi}_{h}+\sin ^{2} \widetilde{\xi}_{h} \cos \Omega_{h}\left(t-t^{\prime}\right)\right] \exp \left[-\left(t-t^{\prime}\right) / T_{R}\right] \frac{d t^{\prime}}{T_{R}},
\end{array}\right.
$$

with $\hbar \Omega_{h}=g_{h} \mu_{B}\left|\vec{B}_{N}^{h}+\vec{B}\right|$. Just after the excitation by the pump pulse $\left(t=0^{+}\right)$, the unexcited hole populations contribute to a spin polarization $\vec{J}_{2}\left(0^{+}\right)$, which can be written as follows:

$$
\left\{\begin{array}{l}
J_{2 x^{\prime}}\left(0^{+}\right)=\cos \left(\frac{\Theta}{2}\right) J_{x^{\prime}}\left(0^{-}\right) \\
J_{2 y^{\prime}}\left(0^{+}\right)=\cos \left(\frac{\Theta}{2}\right) J_{y^{\prime}}\left(0^{-}\right) \\
J_{2 z}\left(0^{+}\right)=\frac{1}{2}\left[1+\cos ^{2}\left(\frac{\Theta}{2}\right)\right] J_{z}\left(0^{-}\right)+\frac{3}{4} \sin ^{2}\left(\frac{\Theta}{2}\right) .
\end{array}\right.
$$

This term $\vec{J}_{2}\left(0^{+}\right)$is the source of a stimulated hole spin $\vec{J}_{2}(t)$, which writes

$$
\begin{aligned}
\vec{J}_{2}(t)= & {\left[\vec{J}_{2}\left(0^{+}\right) \cdot \vec{n}\right] \vec{n}+\left\{\vec{J}_{2}\left(0^{+}\right)-\left[\vec{J}_{2}\left(0^{+}\right) \cdot \vec{n}\right] \vec{n}\right\} \cos \Omega_{h} t } \\
& -\left[\vec{J}_{2}\left(0^{+}\right) \times \vec{n}\right] \sin \Omega_{h},
\end{aligned}
$$

where

$$
\vec{n}=\frac{\vec{B}_{N}^{h}+\vec{B}}{\left|\vec{B}_{N}^{h}+\vec{B}\right|} .
$$

The total hole spin at time $t$ is equal to $\vec{J}(t)=\vec{J}_{1}(t)+\vec{J}_{2}(t)$. The periodicity condition $\vec{J}\left(T_{L}^{-}\right)=\vec{J}\left(0^{-}\right)$leads to a system of linear equations for the initial components $\left[J_{x^{\prime}}\left(0^{-}\right), J_{y^{\prime}}\left(0^{-}\right), J_{z}\left(0^{-}\right)\right]$. Once these variables deduced from the periodicity condition, the time dependence of $\vec{J}(t)$ and $\vec{S}(t)$ can be calculated.

The magnitude and direction of the nuclear field $\vec{B}_{N}^{h}$ are randomly distributed from a QD to another QD and the randomness is described by a Gaussian probability distribution

$$
P\left(\vec{B}_{N}^{h}\right)=\frac{1}{\pi^{3 / 2} \Delta_{\|}^{2} \Delta_{\perp}} \exp \left[-\frac{\left(B_{N x}^{h}\right)^{2}}{\Delta_{\|}^{2}}-\frac{\left(B_{N y}^{h}\right)^{2}}{\Delta_{\|}^{2}}-\frac{\left(B_{N z}^{h}\right)^{2}}{\Delta_{\perp}^{2}}\right],
$$

where $\Delta_{\perp}^{2}\left(\Delta_{\|}^{2}\right)$ is the quadratic average of the out-of-plane (in-plane) nuclear field $\vec{B}_{N}^{h}$,

$$
\Delta_{\perp}^{2}=2\left\langle\left(B_{N z}^{h}\right)^{2}\right\rangle, \quad \Delta_{\|}^{2}=2\left\langle\left(B_{N x}^{h}\right)^{2}\right\rangle=2\left\langle\left(B_{N y}^{h}\right)^{2}\right\rangle .
$$

The experimental measurements being performed on an ensemble of QDs, one gets the total electron spin $S_{z}^{T}(t)$ and hole spin $J_{z}^{T}(t)$ by averaging on the distribution of $\vec{B}_{N}^{h}$ :

$$
\left\{\begin{array}{l}
J_{z}^{T}(t)=\int J_{z}(t) P\left(\vec{B}_{N}^{h}\right) d \vec{B}_{N}^{h} \\
S_{z}^{T}(t)=\int S_{z}(t) P\left(\vec{B}_{N}^{h}\right) d \vec{B}_{N}^{h},
\end{array}\right.
$$

the components of $\vec{B}_{N}^{e}$ being linked to those of $\vec{B}_{N}^{h}$ by Eq. (4).

\section{DISCUSSION}

Figure 3(a) shows the PCD signal for different values of an external magnetic field applied in the Faraday geometry (i.e., for a magnetic field applied along the growth axis of the sample and the propagation direction of the pump beam). For comparison, Fig. 3(a) also contains the PCD signal measured in the absence of an applied magnetic field [see Fig. 1(b)]. The density of excitation is chosen in such a way that the value of the PCD signal obtained for negative delays reaches a maximum. That corresponds to a pump power equal to $200 \mu \mathrm{W}$ for pump and probe beams focused in a circular spot of about $20 \mu \mathrm{m}$ in diameter $\left(60 \mathrm{~W} \mathrm{~cm}^{-2}\right)$.

We observe in Fig. 3(a), as in Fig. 1(b), a fast decay, in the order of hundreds of picoseconds, associated to the presence of photocreated species, and a much slower decay associated 


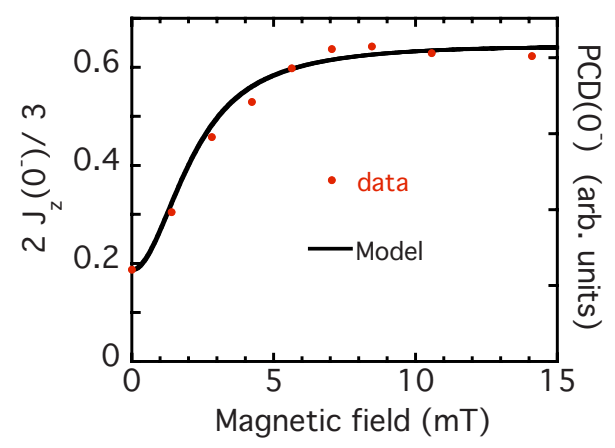

FIG. 4. (Color online) Low-temperature (2 K) magnetic field dependence of the PCD signal at negative delay. Circles represent experimental data. Solid line represents the calculated hole-spin polarization defined in the text as $2 J_{z}\left(0^{-}\right) / 3$. We have used the following values for the calculated curve: $\Theta=1.7,|\beta|=0.42, T_{\Delta}^{e}=550 \mathrm{ps}$, $g_{e}=-0.4, T_{\Delta_{0}}^{h}=4 \mathrm{~ns}$, and a Gaussian probability law centered at $g_{h}=1.35$ with standard deviation equal to 0.5 .

to the hole-spin polarization. The latter signal, as we have already noted, is clearly visible at negative pump-probe delays. Figure 3(a) shows that the amplitude of the PCD signal increases significantly with the increasing amplitude of the applied magnetic field. That is a signature of the mixing of the hole states. Indeed, for a pure hh, the experimental configuration does not induce relaxation through hyperfine coupling ${ }^{18,19}$ and a constant value of the hole-spin polarization should then be found whatever the value of the applied magnetic field.

Circles in Fig. 4 show the experimental data for the PCD signal at negative delays, $\operatorname{PCD}\left(0^{-}\right)$, i.e. the hole-spin polarization at $13 \mathrm{~ns}$, as a function of the applied magnetic field. They follow a quasi-Lorentzian law, with a minimum at zero magnetic field and a maximum and constant value at $B$ $\geq B_{s} \approx 10 \mathrm{mT}$. In recent works, this dependence has been explained in terms of the hole-nuclear interaction. ${ }^{17,20}$ Indeed, in the absence of an external magnetic field, the hole-spin polarization in the QD ensemble decays with time because of the random distribution of the local effective nuclear fields. In an external field $\vec{B}$ applied along the $z$ direction, the effect of the hyperfine interaction on the hole-spin polarization along $z$ can be strongly reduced if the amplitude of this external field is larger than the dispersion $\Delta_{\|}$of the in-plane fluctuations of the nuclear hyperfine field $\vec{B}_{N}^{h}$. From Fig. 4, we obtain a half width at half maximum equal to 2-3 $\mathrm{mT}$.

We have used the model developed in the preceding section to obtain the evolution of the PCD signal as a function of the pump-probe delay and to fit the magnetic field dependence of $\operatorname{PCD}\left(0^{-}\right)$. We have compared and finally fitted the experimental curves to the theoretical ones. In recent experiments, we have determined the values in our sample of the ensemble dephasing time for electrons $T_{\Delta}^{e}$ and the lifetime of the photocreated trion $T_{R}$, which are equal to 500 and $800 \mathrm{ps,}$ respectively. ${ }^{17}$ The longitudinal electron Landé factor has also been determined in a similar manner as described in Ref. $25\left(g_{e}=0.46\right)$. We have used a value of the longitudinal hole Landé factor close to the ones given in the literature for the same kind of samples $\left(g_{h} \approx 1.35\right) . T_{\Delta_{0}}^{h}$ is evaluated to be close to $5 \mathrm{~ns}$ from the hyperfine constants given in Ref. 19. Figure 3(b) shows the calculated $2 J_{z}(t) / 3-2 S_{z}(t)$ curves using $|\beta|$ and $\Theta$ values equal to the final fitting values (see below). We observe that the main features are well reproduced in absence and for small values of the applied magnetic field. But at higher magnetic fields, the theoretical curves do not fit well the experimental results. Particularly, for magnetic field values larger than $B_{s}$, the theoretical curves predict a maximal and quasiconstant value of $2 J_{z}(t) / 3-2 S_{z}(t)$, meanwhile the short-time decay observed at lower magnetic fields does not disappear completely in the corresponding experimental curves.

In order to fit better the experimental curves of the PCD signal, we assume that a hole spin relaxes even when the fluctuations of the nuclear magnetic field are screened by the external field. Hence we consider that mechanisms other than the hyperfine interaction in the framework of the frozen effective nuclear field can take place when this interaction is no more efficient. For example, theoretical studies on the electron-spin relaxation point the existence of a nuclear-spin precession in the Knight field of the electron or dipole-dipole nuclear relaxation. ${ }^{9,10}$ Concerning theoretical studies on the hole-spin relaxation, another mechanism has been described recently: the spin-orbit-mediated phonon scattering between Zeeman levels. ${ }^{26,27}$

To modelize in a phenomenological way the existence of a hole-spin relaxation mechanism which exists beyond the one due to the hyperfine coupling, we modified Eqs. (9) and (11) and assumed an exponentially damped precession of the holes around the total magnetic field (nuclear plus external) with a characteristic time $T_{1}$. Figure 3(c) shows the temporal behavior of the calculated $2 J_{z}(t) / 3-2 S_{z}(t)$ curves for different magnetic fields when $T_{1}$ is taken to be $0.3 \mu \mathrm{s}$. Now, the short-living component observed for applied magnetic fields close to $B_{s}$ is well reproduced in the calculated curves.

We can also remark that the amplitude of variation of the PCD signal at negative delays as a function of the applied magnetic field depends on the values of $|\beta|$. Figure 5(a) shows the calculated $2 J_{z}\left(0^{-}\right) / 3$ dependence on the external magnetic field, at $\Theta=\pi$ and for different $|\beta|$ values. For the ensemble of these curves, $T_{1}$ is taken to be infinite. Figure 5(b) shows the same ensemble of calculated curves for a finite value of $T_{1}\left(T_{1}=0.3 \mu \mathrm{s}\right)$. We observe that, under these conditions, the ratio between the maximum and the minimum of each curve is fixed by the value of $|\beta|$, whatever the value of $T_{1}$. This ratio is equal to 1 for pure hh states and, for mixed holes, increases as $|\beta|$ increases. We conclude that the ratio, at negative delay, between the PCD signal at $B=B_{s}$ to the PCD signal at $B=0$, which is obtained for a power density of the pump beam close to the conditions of a maximum of PCD signal, is mainly fixed by the value of $|\beta|$, i.e. the mixed character of the hole states.

Figures 5(a) and 5(b) also show that the maximum value of the hole-spin polarization at $13 \mathrm{~ns}, 2 J_{z}\left(0^{-}\right) / 3$, depends not only on $|\beta|$ but also on $T_{1}$. That is why further experimental work on ultraslow hole-spin dynamics is required.

To summarize, once the experimental pump power density has been optimized to obtain a maximum of $\operatorname{PCD}\left(0^{-}\right)$ signal, the procedure to fit the PCD curves can be described simply and splits in several steps. First, from the ratio of the 


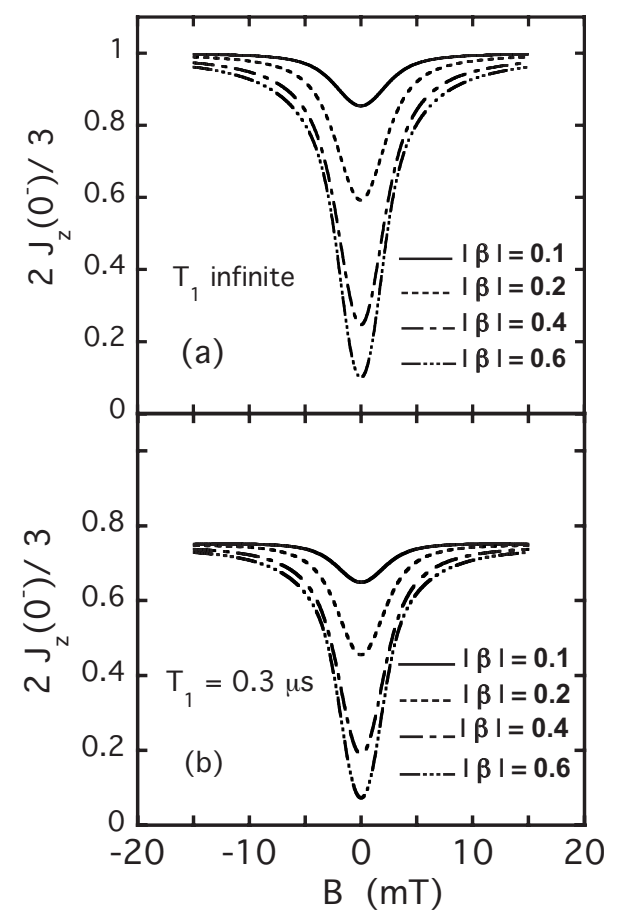

FIG. 5. Calculated magnetic field dependence of the hole-spin polarization at $13 \mathrm{~ns}, 2 J_{z}\left(0^{-}\right) / 3$, for $\Theta=\pi$ and for different values of the hole mixing $|\beta|$. (a) $T_{1}$ is taken to be infinite. (b) $T_{1}$ takes a finite value: $T_{1}=0.3 \mu \mathrm{s}$. We have used a Gaussian probability law centered at $g_{h}=1.35$ with standard deviation equal to 0.5 .

$\operatorname{PCD}\left(0^{-}\right)$value at $B \approx B_{s}$ to the one at $B=0$, we obtain a first estimation of the $|\beta|$ value. Second, by fitting the PCD temporal curves at small magnetic fields, we obtain a more precise estimation of the $|\beta|$ and $\Theta$ values. Third, the $T_{1}$ value is mainly determined by the fitting of the PCD curves at $B$ $\approx B_{s}$. Finally, the whole set of parameters is optimized by fitting the temporal dependence of the ensemble of PCD curves obtained for different values of the applied magnetic field. Circles of Fig. 4 show the magnetic field dependence of the PCD signal at negative pump-probe delay and solid line represents the fitting curve obtained for $|\beta|=0.42$ and $\Theta=1.7$. Figure 6 shows the calculated curves for the same parameters as those obtained in Fig. 3(c), including $T_{1}$ $=0.3 \mu \mathrm{s}$. We observe that the theoretical curves fit very well the experimental data and in particular the short-time com-

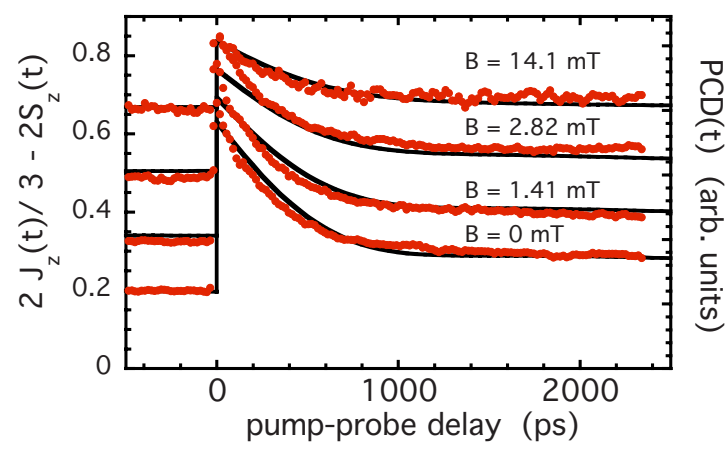

FIG. 6. (Color online) Low-temperature (2 K) PCD signals for different values of the external Faraday magnetic field $\vec{B}$. We show a selection of four experimental curves among those shown in Fig. 3(a) (circles). We have used the following values in the calculated curves: $g_{h}=1.35, g_{e}=-0.4, T_{R}=780 \mathrm{ps}, T_{\Delta}^{e}=550 \mathrm{ps}, \Theta=1.7,|\beta|$ $=0.42$, and $T_{\Delta_{0}}^{h}=4 \mathrm{~ns}$ (solid lines). The good agreement of the calculated curves with experiment, especially for the short-time interval in high external fields, is obtained with a supplementary relaxation time $T_{1}=0.3 \mu$ s of the hole-spin polarization.

ponent of the PCD signal for magnetic fields which screen the hyperfine interaction.

In conclusion, we have measured the PCD signal in $p$-doped InAs/GaAs QDs as a function of an applied magnetic field in a Faraday geometry at low temperature. The amplitude of the PCD signal increases and saturates with increasing magnetic fields. This behavior is due to the screening of the hole-nuclear hyperfine interaction and is a signature of the heavy- and light-hole mixing. We have modelized the interconnected dynamics of the photoelectron spin and the resident hole spin and compared the theoretical curves to the experimental PCD data. We verified, in this way, that the hole-nuclear hyperfine coupling is 10-20 times smaller than the electron-nuclear coupling. As predicted in recent theoretical works, we need an extra hole-spin relaxation time, slower than the hyperfine-induced dephasing time and which is in the order of $1 \mu \mathrm{s}$. Further experimental investigation on ultraslow hole-spin dynamics is required.

\section{ACKNOWLEDGMENTS}

One of us (B.E.) thanks the C'Nano-IdF for its financial support.
${ }^{1}$ M. Chamarro, F. Bernardot, and C. Testelin, J. Phys.: Condens. Matter 19, 445007 (2007).

${ }^{2}$ M. Atature, J. Dreiser, A. Badolato, A. Hogele, K. Karrai, and A. Imamoglu, Science 312, 551 (2006).

${ }^{3}$ X. Xu, Y. Wu, B. Sun, Q. Huang, J. Cheng, D. G. Steel, A. S. Bracker, D. Gammon, C. Emary, and L. J. Sham, Phys. Rev. Lett. 99, 097401 (2007).

${ }^{4}$ A. Greilich, R. Oulton, E. A. Zhukov, I. A. Yugova, D. R. Yakovlev, M. Bayer, A. Shabaev, Al. L. Efros, I. A. Merkulov, V. Stavarache, D. Reuter, and A. Wieck, Phys. Rev. Lett. 96, 227401 (2006).
${ }^{5}$ J. Berezovsky, O. Gywat, F. Meier, D. Battaglia, X. Peng, and D. D. Awschalom, Nat. Phys. 2, 831 (2006).

${ }^{6}$ D. Press, T. D. Ladd, B. Zhang, and Y. Yamamoto, Nature (London) 456, 218 (2008).

${ }^{7}$ A. Greilich, S. E. Economou, S. Spatzek, D. R. Yakovlev, D. Reuter, A. D. Wieck, T. L. Reinecke, and M. Bayer, Nat. Phys. 5, 262 (2009).

${ }^{8}$ E. D. Kim, K. Smirl, Y. Wu, A. Amo, X. Xu, D. G. Steel, A. S. Bracker, D. Gammon, and L. J. Sham, arXiv:0905.0297 (unpublished).

${ }^{9}$ A. V. Khaetskii, D. Loss, and L. Glazman, Phys. Rev. Lett. 88, 
186802 (2002); Phys. Rev. B 67, 195329 (2003).

${ }^{10}$ I. A. Merkulov, Al. L. Efros, and M. Rosen, Phys. Rev. B 65 , 205309 (2002).

${ }^{11}$ P.-F. Braun, X. Marie, L. Lombez, B. Urbaszek, T. Amand, P. Renucci, V. K. Kalevich, K. V. Kavokin, O. Krebs, P. Voisin, and Y. Masumoto, Phys. Rev. Lett. 94, 116601 (2005).

${ }^{12}$ A. C. Johnson, J. R. Petta, J. M. Taylor, A. Yacoby, M. D. Lukin, C. M. Marcus, M. P. Hanson, and A. C. Gossard, Nature (London) 435, 925 (2005).

${ }^{13}$ D. Heiss, S. Schaeck, H. Huebl, M. Bichler, G. Abstreiter, J. J. Finley, D. V. Bulaev, and D. Loss, Phys. Rev. B 76, 241306(R) (2007).

${ }^{14}$ B. D. Gerardot, D. Brunner, P. A. Dalgarno, P. Ohberg, S. Seidl, M. Kroner, K. Karrai, N. G. Stoltz, P. M. Petroff, and R. J. Warburton, Nature (London) 451, 441 (2008).

${ }^{15}$ A. J. Ramsay, S. J. Boyle, R. S. Kolodka, J. B. B. Oliveira, J. Skiba-Szymanska, H. Y. Liu, M. Hopkinson, A. M. Fox, and M. S. Skolnick, Phys. Rev. Lett. 100, 197401 (2008).

${ }^{16}$ D. Heiss, V. Jovanov, M. Bichler, G. Abstreiter, and J. J. Finley, Phys. Rev. B 77, 235442 (2008).

${ }^{17}$ B. Eble, C. Testelin, P. Desfonds, F. Bernardot, A. Balocchi, T. Amand, A. Miard, A. Lemaître, X. Marie, and M. Chamarro, Phys. Rev. Lett. 102, 146601 (2009).

${ }^{18}$ J. Fischer, W. A. Coish, D. V. Bulaev, and D. Loss, Phys. Rev. B 78, 155329 (2008).

${ }^{19}$ C. Testelin, F. Bernardot, B. Eble, and M. Chamarro, Phys. Rev. B 79, 195440 (2009).

${ }^{20}$ M. V. Gurudev Dutt, J. Cheng, B. Li, X. Xu, X. Li, P. R. Berman, D. G. Steel, A. S. Bracker, D. Gammon, S. E. Economou, R.-B. Liu, and L. J. Sham, Phys. Rev. Lett. 94, 227403 (2005).

${ }^{21}$ A. Shabaev, Al. L. Efros, D. Gammon, and I. A. Merkulov, Phys. Rev. B 68, 201305(R) (2003).
${ }^{22}$ A. Greilich, D. R. Yakovlev, A. Shabaev, Al. L. Efros, I. A. Yugova, R. Oulton, V. Stavarache, D. Reuter, A. Wieck, and M. Bayer, Science 313, 341 (2006).

${ }^{23}$ I. A. Yugova, A. A. Sokolova, D. R. Yakovlev, A. Greilich, D. Reuter, A. D. Wieck, and M. Bayer, Phys. Rev. Lett. 102, 167402 (2009).

${ }^{24}$ We have studied the photoluminescence and PCD signals of a set of samples with different nominal $p$-doping contents (from 0 to $2 \times 10^{11} \mathrm{~cm}^{-2}$ ). By an analysis and a comparative study of the results, we are able to determine the growth conditions leading to an optimum doping close to one hole per QD. As shown in Ref. 28, the undoped QDs show linear photoinduced dichroism due to the linear symmetry of the neutral excitonic states with a very short-living PCD signal associated to coherence induced by the pump and called dynamical photoinduced dichroism. We have observed that this short-time contribution is vanishing for the sample discussed in the paper $\left(2 \times 10^{11} \mathrm{~cm}^{-2}\right.$ doping $)$, which permits to assume a high concentration of singly charged QD, as discussed in Ref. 29.

${ }^{25}$ L. Lombez, P. F. Braun, X. Marie, P. Renucci, B. Urbaszek, T. Amand, O. Krebs, and P. Voisin, Phys. Rev. B 75, 195314 (2007).

${ }^{26}$ D. V. Bulaev and D. Loss, Phys. Rev. Lett. 95, 076805 (2005).

${ }^{27}$ M. Trif, P. Simon, and D. Loss, Phys. Rev. Lett. 103, 106601 (2009).

${ }^{28}$ F. Bernardot, E. Aubry, J. Tribollet, C. Testelin, M. Chamarro, L. Lombez, P.-F. Braun, X. Marie, T. Amand, and J.-M. Gérard, Phys. Rev. B 73, 085301 (2006).

${ }^{29}$ A. I. Tartakovskii, M. N. Makhonin, J. Cahill, D. M. Whittaker, J.-P. R. Wells, A. M. Fox, D. J. Mowbray, M. S. Skolnick, M. J. Steer, K. M. Groom, and M. Hopkinson, Appl. Phys. Lett. 85, 2226 (2004). 